\title{
Approximations of the solutions of a stochastic differential equation using Dirichlet process mixtures and Gaussian mixtures
}

\author{
Saba Infante ${ }^{1, *}$, César Luna ${ }^{1}$, Luis Sánchez ${ }^{2}$, Aracelis Hernández ${ }^{1}$ \\ ${ }^{1}$ Department of Mathematics, Faculty of Science and Technology, University of Carabobo, Venezuela \\ ${ }^{2}$ Department of Mathematics, Faculty of Education. University of Carabobo, Venezuela
}

(Received: 31 July 2016; Accepted: 14 November 2016)

\begin{abstract}
Stochastic differential equations arise in a variety of contexts. There are many techniques for approximation of the solutions of these equations that include statistical methods, numerical methods, and other approximations. This article implements a parametric and a nonparametric method to approximate the probability density of the solutions of stochastic differential equation from observations of a discrete dynamic system. To achieve these objectives, mixtures of Dirichlet process and Gaussian mixtures are considered. The methodology uses computational techniques based on Gaussian mixtures filters, nonparametric particle filters and Gaussian particle filters to establish the relationship between the theoretical processes of unobserved and observed states. The approximations obtained by this proposal are attractive because the hierarchical structures used for modeling are flexible, easy to interpret and computationally feasible. The methodology is illustrated by means of two problems: the synthetic Lorenz model and a real model of rainfall. Experiments show that the proposed filters provide satisfactory results, demonstrating that the algorithms work well in the context of processes with nonlinear dynamics which require the joint estimation of states and parameters. The estimated measure of goodness of fit validates the estimation quality of the algorithms.
\end{abstract}

Keywords Gaussian mixtures filter; Nonparametric particle filter; Gaussian particle filter

AMS 2010 subject classifications 62L10, 62L20

DOI: $10.19139 /$ soic.v4i4.242

\section{Introduction}

Stochastic differential equations are used in many applications related to basic science such as modeling of biological, chemical, physical, environmental, engineering, economics and finance processes among others ([36]). A stochastic differential equation describes the time evolution of the dynamics of a state vector, based on physical observations from a real system obtained with errors. In practice, the interest consists in estimating the states or parameters of the dynamic system. One of the difficulties commonly encountered in the estimation of parameters in a stochastic differential equation is that the transition density of the equation can not be evaluated in closed form. There are many estimation methods that are based on the maximum simulated Likelihood methods ([64]; [65]; [53]; [22]; [1]; [49] and [21]). Another standard method is to approximate the solutions using the Markov Chain Monte Carlo (MCMC) algorithm ([44]; [27]; [13]; [66] and [67]). It is also possible to approximate solutions by using numerical methods ([23] and [26]). There are others methods based on Hermite expansions ([1]); Taylor approximations ([25]); filtering Theory ([25]; [43] and [24]); approaches that use algorithms based on the integration of Gaussian quadratures and sigma point methods ([28]; [29]; [60] and [23]); adaptive MCMC algorithms based on numerical integration methods recently have been used to approximate in the context of

\footnotetext{
${ }^{*}$ Correspondence to: Saba Infante (Email: sinfante@uc.edu.ve). Department of Mathematics, University of Carabobo, Venezuela
}

ISSN 2310-5070 (online) ISSN 2311-004X (print)

Copyright (C) 2016 International Academic Press 
nonlinear stochastic differential equations, for example [60] and [5] have used an approach based on a Gaussian process to model the time evolution of the solution of a general stochastic differential equation, the methodology uses data assimilation techniques ([31]). Recently [57] introduced a nonparametric method to estimate the function of drift in a stochastic differential equation where a measure of random probability was considered to model the drift and a Expectation-Maximization (EM) algorithm was developed to try to establish a link between unobserved and observed states.

This paper aims to approximate the solutions of a stochastic differential equation by using the Gaussian mixture distribution model, Dirichlet process mixture models, together with a non-parametric density estimation algorithm and three sequential filters. The most representative references about the Gaussian mixture distribution model are [61]; [3]; [68] and [2]. Dirichlet processes were treated in [16], and they have been identified in the literature as a useful tool for estimating densities in the field of non-parametric models. The idea of using Dirichlet process is not new, and has been considered in previous works such as [15]; [46]; [42]; [45]; [70] among others.

The rest of article is summarized as follows: Section 2 presents the formulation of the problem; Section 3 contains the description of the filtering algorithms defined to approximate a probability density of the solutions; In Section 4 , the results obtained for two different examples are shown, and lastly, Section 5 contains a final discussion and conclusions.

\section{Formulation of the problem}

This article proposes to use approximations, obtained by nonlinear filters, to estimate the solution of an It $\hat{o}$ stochastic differential equation (SDE) given by:

$$
d \mathbf{x}_{k}=f\left(\mathbf{x}_{k}, k\right) d k+L\left(\mathbf{x}_{k}, k\right) d \mathbf{B}_{k}
$$

where $\mathbf{x}_{k} \in \mathbb{R}^{n}$ is a vector of stochastic states in a continuous-time, $\mathbf{x}_{k_{0}}$ is a stochastic initial condition satisfying the following condition $\mathbb{E}\left(\left|\mathbf{x}_{k_{0}}^{2}\right|\right)<\infty ;\left\{\mathbf{B}_{k}: k \geq 0\right\}$ is a $m$ - dimensional vector of a standard Brownian motion; $F:\left[k_{0}, T\right] \times \mathbb{R}^{n} \times \mathbb{R}^{d} \rightarrow \mathbb{R}^{n}$ is a known nonlinear drift function, and $L:\left[k_{0}, T\right] \times \mathbb{R}^{n} \times \mathbb{R}^{d} \rightarrow \mathbb{R}^{n} \times \mathbb{R}^{m}$ is a diffusion matrix that satisfies sufficient regularity conditions to ensure the existence of strong solutions ([52]). Theoretically the system is imperfectly observed through a process of continuous observation $\mathbf{y}_{k} \in \mathbb{R}^{m}$ which is related to the process $\mathbf{x}_{k}$ by the equation:

$$
\mathbf{y}_{k}=\int_{0}^{k} h\left(\mathbf{x}_{s}, s, \theta\right) d s+\sqrt{\mathbf{D}_{k}} \mathbf{W}_{k}
$$

where $h: \mathbb{R}^{n} \times \mathbb{R} \rightarrow \mathbb{R}$ is a known function, $\left\{\mathbf{W}_{k}: k \geq 0\right\}$ is a vector of a standard Brownian motion, which is independent of $\mathbf{B}_{k}, \mathbf{D}_{k}$ is a matrix of diffusion, and the initial state $\mathbf{x}_{0}$.

When the dynamic system in continuous time is approximated by a discrete system, the derivatives in continuous time are approximated by difference equations in discrete time, which can be expressed in the form of a space state model, as follows:

$$
\begin{aligned}
\mathbf{x}_{k} & =\mathbf{M}_{k}\left(\mathbf{x}_{k-1}, \mathbf{u}_{k}\right) \\
\mathbf{y}_{k} & =\mathbf{H}_{k}\left(\mathbf{x}_{k}\right)+\mathbf{v}_{k} \\
\mathbf{u}_{k} & \sim F(., .)
\end{aligned}
$$

The equation given in (3) represents a dynamic system, where $\mathbf{x}_{k}$ denotes the vector of unknown states in a time $t=k, \mathbf{u}_{k}$ is a random error state estimation, $\mathbf{M}_{k}$ is an operator that maps the space transition state within the state space. The equation given in (4) represents the observed system, where $\mathbf{H}_{k}$ is an operator that maps the states within the space of observations in time $t=k, \mathbf{y}_{k}$ is the vector of observations, and $\mathbf{v}_{k}$ is a vector of random errors of observation. In the given equation (5), $F(.,$.$) is a function of unknown probability distribution. The$ unobserved solutions $x_{k}$ are assumed to be modeled by a Markov process of first order, with an initial distribution 
function $p\left(x_{0}\right)$, and transition equation $p\left(x_{k} \mid x_{k-1}\right)$ given by the model defined in equation (3). Each observation $y_{k}$ is conditionally assumed to be independent given the state $x_{k}$, where $p\left(y_{k} \mid x_{k}\right)$ is obtained from equation (4). The model is summarized in three steps: first an initial seed $x_{0} \sim p\left(x_{0}\right)$ is generated; then $x_{k} \sim p\left(x_{k} \mid x_{k-1}\right)$ is predicted; and finally $y_{k} \sim p\left(y_{k} \mid x_{k}\right)$ is updated. The general objective is to estimate the unknown states $\mathbf{x}_{k}=$ $x_{0: k}=\left(x_{0}, \ldots, x_{k}\right)$, based on measurements obtained from the observation process $\mathbf{y}_{k}=y_{1: k}=\left(y_{1}, \ldots, y_{k}\right)$; the main interest is in estimating the filtering distribution $p\left(x_{k} \mid y_{1: k}\right)$, the predictive distribution $p\left(x_{k+1} \mid y_{1: k}\right)$, and posterior mean and covariance. The recursive estimation in time $k$, is performed by the following procedure:

- Step 1: The filtering distribution:

$$
p\left(x_{k} \mid y_{1: k}\right)=\int p\left(x_{k} \mid x_{k-1}\right) p\left(x_{k-1} \mid y_{1: k-1}\right) d x_{k-1}
$$

- Step 2: The predictive distribution:

$$
p\left(x_{k+1} \mid y_{1: k}\right)=\int p\left(x_{k+1} \mid x_{k}\right) p\left(x_{k} \mid y_{1: k-1}\right) d x_{k}
$$

- Step 3: The posterior predictive expectation:

$$
m_{k \mid k}=\mathbb{E}\left(x_{k} \mid y_{1: k}\right)=\int x_{k} p\left(x_{k} \mid y_{1: k}\right) d x_{k}
$$

- Step 4: The posterior predictive covariance:

$$
P_{k \mid k}=\mathbb{E}\left[\left(x_{k}-m_{k \mid k}\right)\left(x_{k}-m_{k \mid k}\right)^{T}\right]=\int\left(x_{k}-m_{k \mid k}\right)\left(x_{k}-m_{k \mid k}\right)^{T} p\left(x_{k} \mid y_{1: k}\right) d x_{k}
$$

Assuming that $\mathbf{v}_{k}$ is distributed according to a known probability density function $\mathbf{v}_{k} \sim N\left(\mathbf{0}, \mathbf{R}_{k}\right)$ with fixed but unknown parameters, $\mathbf{R}_{k}$ is to be estimated. If, in addition, the errors $\mathbf{u}_{k}$ do not have a known distribution, that is $\mathbf{u}_{k} \sim F(.,$.$) , where F(.,$.$) is an unknown function, then it is necessary to estimate the probability distribution$ function $F(.,$.$) .$

At some time $t=k$, we can estimate the posterior distribution joint $p\left(\mathbf{x}_{0: k} \mid \mathbf{y}_{1: k}\right)$ using the Bayes Theorem, as follows:

$$
p\left(x_{0: k} \mid y_{1: k}\right)=\frac{p\left(y_{1: k} \mid x_{0: k}\right) p\left(x_{0: k}\right)}{\int p\left(y_{1: k} \mid x_{0: k}\right) p\left(x_{0: k}\right) d x_{0: k}}
$$

It is possible to obtain a recursive formula to estimate the joint density $p\left(x_{0: k} \mid y_{1: k}\right)$ :

$$
p\left(x_{0: k+1} \mid y_{1: k+1}\right)=p\left(x_{0: k} \mid y_{1: k}\right) \frac{p\left(y_{k+1} \mid x_{k+1}\right) p\left(x_{k+1} \mid x_{k}\right)}{p\left(y_{k+1} \mid y_{1: k}\right)}
$$

where the filtering distribution $p\left(x_{k} \mid y_{1: k}\right)$ is estimated by the following recursive Bayesian filter:

1. It is initialized with a prior distribution $p\left(x_{0}\right)$.

2. For $k=1, \ldots, N$ run the following:

- Prediction step: the state $x_{k}$ is predicted, which can be calculated:

- In the discrete case: The Chapman-Kolmogorov equation:

$$
p\left(x_{k} \mid y_{1: k-1}\right)=\int p\left(x_{k} \mid x_{k-1}\right) p\left(x_{k-1} \mid y_{1: k-1}\right) d x_{k-1}
$$

- In the continuous case: The Fokker-Planck-Kolmogorov equation is integrated:

$$
\frac{\partial p(x, k)}{\partial k}=-\sum_{i=1}^{n} \frac{\partial}{\partial x_{i}}\left[f_{i} p(x, k)\right]+\frac{1}{2} \sum_{i=1}^{n} \sum_{j=1}^{n} \frac{\partial^{2}}{\partial x_{i} \partial x_{j}}\left\{\left[L Q L^{T}\right]_{i j} p(x, k)\right\}
$$

where: $p(x, k)=p\left(x_{k} \mid y_{1: k-1}\right), f_{i}=f_{i}(x, k)$, y $L=L(x, k)$. 
- Update step: given the observation $y_{k}$, the predictive distribution is updated by:

$$
p\left(x_{k} \mid y_{1: k}\right)=\frac{p\left(y_{k} \mid x_{k}\right) p\left(x_{k} \mid y_{1: k-1}\right)}{\int p\left(y_{k} \mid x_{k}\right) p\left(x_{k} \mid y_{1: k-1}\right) d x_{k}}
$$

Given the difficulty of calculating the integrals involved in (12) and (14); a way to solve the situation is to use computational techniques to approximate the posterior distribution. This article propose three recursive algorithms to achieve this goal.

A particular problem to be solved in this work is to estimate a probability density using a nonparametric Bayesian method involving an endless mix model as considered in [42] and [56]; but in the context of stochastic differential equations. We propose to use the following hierarchical structure to flexibly approximate the probability density of the solutions of the equation given in (1):

$$
\begin{gathered}
x_{k-1} \mid \theta_{k} \sim f\left(x_{k-1} \mid \theta_{k}\right) \quad ; \quad k=1, \ldots, t \\
\theta_{k}(.,) \sim \mathbb{G}(., .) \\
\mathbb{G}(., .) \sim D P\left(\alpha, \mathbb{G}_{0}\right) \\
x_{k}=M_{k}\left(x_{k-1}, u_{k}\right)=\sum_{i=1}^{P} \omega_{i} f\left(x_{i-1} \mid \theta_{i}\right) \quad, \quad \sum_{i=1}^{n} \omega_{i}=1 \\
x_{k+1} \mid x_{k} \sim N(\mu, \Sigma) \\
y_{k} \mid x_{k+1} \sim N\left(H_{k}\left(x_{k+1}\right), R_{k}\right)
\end{gathered}
$$

where $D P$ is a Dirichlet processes and:

$$
\begin{gathered}
\theta_{k}=\left(\mu_{k}, \Sigma_{k}\right) \quad ; \quad f\left(x_{k} \mid \mu_{k}, \Sigma_{k}\right)=N\left(\mu_{k}, \Sigma_{k}\right) \\
\mathbb{G}\left(\mu_{k}, \Sigma_{k}\right)=\mathbb{G}\left(\mu_{k} \mid \Sigma_{k}\right) \mathbb{G}\left(\Sigma_{k}\right)=N\left(\lambda, \frac{\Sigma_{k}}{\tau}\right) I W\left(\Sigma_{0}, \nu\right) \\
\mu=\sum_{i=1}^{n} \omega_{i} \mu_{i} \quad ; \quad \Sigma=\sum_{i=1}^{n} \omega_{i}\left[\Sigma_{i}+\left(\mu_{i}-\mu\right)\left(\mu_{i}-\mu\right)^{T}\right]
\end{gathered}
$$

\section{Filtering algorithms to approximate a density}

Here, three filtering algorithms are proposed to approximate the solutions of stochastic differential equation: Gaussian Mixtures Filter (GMF), Nonparametric Particle Filtering (NPF), and Gaussian Particle Filter (GPF). 


\subsection{Nonparametric density estimation}

The problem of estimating a density using a nonparametric method consists of the following, data $x_{1}, \ldots, x_{n}$ are assumed to be an interchangeable sequence of independent observations taken according to a unknown probability density function $F$, that is:

$$
x_{i} \sim F(., .) \quad ; \quad i=1, \ldots, n
$$

The $x_{i}$ can be scalars or vectors. Traditional methods of parametric statistics, consider probability models that are indexed by unknown finite dimensional parameters, such as the mean and variance, which must be estimated. In contrast, Bayesian nonparametric methods consider a prior probability model $p(F)$ for the unknown distribution function $F$, where $F$ is a function in a space of infinite dimension. This requires the definition of a measure of random probability of a collection of distributions functions which should have certain properties such as having a large support and the condition that the resulting posterior distribution must be analytically tractable ([16]).

The $D P$ ([16] and [62]), are an important Bayesian nonparametric modeling tool. A random probability distribution $F$ is generated by a $D P$ if for any partition $A_{1}, \ldots, A_{k}$ of sample space $\Omega$, the vector:

$$
\left(F\left(A_{1}\right), \ldots, F\left(A_{k}\right)\right) \sim \operatorname{Dir}\left(\alpha F_{0}\left(A_{1}\right), \ldots, \alpha F_{0}\left(A_{k}\right)\right)
$$

where $\operatorname{Dir}\left(\alpha_{1}, \ldots, \alpha_{k}\right)$ denotes the Dirichlet distributions; $F_{0}($.$) is a base measure of random probability defining$ the expected value, $\mathbb{E}(F)=F_{0}$ or equivalently $\mathbb{E}(F(A))=F_{0}(A)$ for any $A \subset \Omega$, and $\alpha$ is a precision parameter that defines the variance. The motivation to use the $D P$ is that, in this approach, updating the posterior distribution is a simple process. That is:

$$
\begin{aligned}
& x_{1}, \ldots, x_{n} \mid F \sim F \\
& F \sim \operatorname{Dir}\left(F_{0}, \alpha\right) \\
& F \mid x_{1}, \ldots, x_{n} \sim \operatorname{Dir}\left(\alpha+n, F_{0}+\sum_{i=1}^{n} \delta_{x_{i}}\right)
\end{aligned}
$$

where $\delta_{x_{i}}$ denotes the Dirac delta.

In a Dirichlet mixture process model (DPM), samples $x_{i}$, for $i=1, \ldots, n$, are taken from a component of the mixture parametrized by $\theta_{i} \in \Theta$, where $\theta_{i}$ is a latent variable. This auxiliary variable is generated by an unknown distribution $\mathbb{G}$, which represents a measure of random probability distributed according to a prior distribution (a distribution on the set of probability distributions). That is, the DPM is hierarchically defined as follows:

$$
\begin{aligned}
& x_{i} \mid \theta_{i} \sim f\left(x_{i} \mid \theta_{i}\right) \quad ; \quad i=1, \ldots, n \\
& \theta_{i} \mid \mathbb{G} \sim \mathbb{G} \\
& \mathbb{G} \sim D P\left(\alpha, \mathbb{G}_{0}\right)
\end{aligned}
$$

where the mixing distribution $\mathbb{G}$ is a $D P$ with concentration parameter $\alpha$, and distribution base $\mathbb{G}_{0}$. If integrated on random measure $\mathbb{G}$, we have a non-parametric model to estimate $F$, as follows:

$$
F(x)=\int_{\theta} f(x \mid \theta) d \mathbb{G}(\theta) \quad ; \quad \mathbb{G} \sim D P\left(\alpha, \mathbb{G}_{0}\right)
$$

There are two basic representations of $D P$, known as Polya urn scheme ([7]), and a prior Stick-breaking scheme ([62] and [54]). The scheme of Polya urn, assumes that the joint distribution of $\theta_{1}, \ldots, \theta_{n}$, can be expressed as follows:

$$
\begin{aligned}
& \theta_{1} \sim \mathbb{G}_{0}\left(\theta_{1}\right) \\
& \theta_{i} \mid \theta_{1}, \ldots, \theta_{i-1} \sim \frac{\alpha \mathbb{G}_{0}+\sum_{l=1}^{i-1} \delta_{\theta_{i} \mid \theta_{l}}}{\alpha+i-1}
\end{aligned}
$$


where $\delta_{\theta_{i} \mid \theta_{l}}$ is a point mass $\theta_{l}$. When $\alpha \rightarrow 0$, all the $\theta_{i}=\theta_{1} \sim \mathbb{G}_{0}\left(\theta_{1}\right)$, and when $\alpha \rightarrow \infty, \theta_{i} \sim \mathbb{G}_{0}\left(\theta_{1}\right)$. Moreover, as the $\theta_{i}$ are exchangeable the Polya urn scheme can be written as:

$$
\theta_{i} \mid \theta_{-i} \sim \frac{\alpha \mathbb{G}_{0}+\sum_{i \neq l} \delta_{\theta_{i} \mid \theta_{l}}}{\alpha+n-1}
$$

where $\theta_{-i}$ represents the $\left\{\theta_{l}: i \neq l\right\}$.

A strategy for representation the $D P$, using the scheme called Stick-breaking, is to write the measure $\mathbb{G}$ explicitly as an infinite sum of sums of atomic measures [62]. Embodiments of a $D P$ are expressed as follows:

$$
\mathbb{G}=\sum_{k=1}^{\infty} \omega_{k} \delta_{\theta_{k}}
$$

where, $\delta_{\theta_{k}}$ denotes the measure of the Dirac delta located at $\theta_{k}$ and

$$
\begin{aligned}
& \theta_{k} \sim \mathbb{G}_{0} \\
& \omega_{k}=\beta_{k} \prod_{j=1}^{k-1}\left(1-\beta_{j}\right) \\
& \beta_{k} \sim \operatorname{Beta}(1, \alpha)
\end{aligned}
$$

The underlying random measure $\mathbb{G}$ is discrete with probability one. Using equation (19), a flexible a priori model used to estimate the unknown distribution $F$ is obtained:

$$
F(x)=\sum_{k=1}^{\infty} \omega_{k} f\left(x \mid \theta_{k}\right)
$$

A recursive form to estimate the unknown probability density in nonparametric form is given as follow:

\section{Algorithm 1: Nonparametric algorithm to estimate a density}

- Step 1: Simulate:

$$
x_{k-1} \mid \mu_{k}, \Sigma_{k} \sim N\left(\mu_{k}, \Sigma_{k}\right)
$$

- Step 2: Simulate:

$$
\left(\mu_{k}, \Sigma_{k}\right) \sim N I W\left(\lambda, \nu, \Sigma_{0}, \gamma_{0}\right)
$$

where:

- The conditional mean is obtained by:

$$
\mu_{k} \mid \Sigma_{k}, \lambda, \tau \sim N\left(\lambda, \frac{\Sigma_{k}}{\tau}\right)
$$

- The conditional variance is obtained by:

$$
\Sigma_{k} \mid \Sigma_{0}, \nu \sim I W\left(\Sigma_{0}, \nu\right)
$$

- Step 3: Calculate:

$$
x_{k}=M_{k}\left(x_{k-1}, u_{k-1}\right)=\sum_{i=1}^{n} \omega_{i} N\left(\mu_{i}, \Sigma_{i}\right) \quad ; \quad \sum_{i=1}^{n} \omega_{i}=1
$$

- Step 4: Conditioned states are generated $x_{k+1} \mid x_{k}$, as follows:

$$
x_{k+1} \mid x_{k} \sim N(\mu, \Sigma)
$$

- Step 5: The observations of the states are generated $y_{k} \mid x_{k+1}$ :

$$
y_{k} \mid x_{k+1} \sim N\left(\mathcal{H}\left(x_{k+1}\right), R\right)
$$

where $I W$ denotes Inverse Wishart distribution, and $N I W(., ., .,$.$) denotes a Normal Inverse Wishart.$ 


\subsection{Gaussian mixtures Filter (GMF)}

If we assume that the observation model $y_{k}=H_{k}\left(x_{k}, v_{k}\right)=H x_{k}+v_{k}$ is linear, where $v_{k} \sim N(0, R)$ and also assume that the a priori model of a state $x$ is approximated by $p(x)$, and the likelihood is approximated by $p(y \mid x)$, where:

$$
p(x)=\sum_{i=1}^{n} \omega_{i} N(\mu, \Sigma) \quad ; \quad \sum_{i=1}^{n} \omega_{i}=1
$$

and

$$
p(y \mid x)=\sum_{j=1}^{m} \beta_{j} N(H x, R) \quad ; \quad \sum_{j=1}^{m} \beta_{j}=1
$$

Then using equations (32) and (33), the posterior distribution is a Gaussian mixture given by:

$$
\begin{aligned}
p(x \mid y) & =\frac{p(y \mid x) p(x)}{\int p(y \mid x) p(x) d x} \\
& =\frac{\sum_{j=1}^{m} \beta_{j} N(y ; H x, R) \sum_{i=1}^{n} \omega_{i} N(x ; \mu, \Sigma)}{\int \sum_{j=1}^{m} \beta_{j} N(y ; H x, R) \sum_{i=1}^{n} \omega_{i} N(x ; \mu, \Sigma) d x} \\
& =\frac{\sum_{j=1}^{m} \sum_{i=1}^{n} \omega_{i} \beta_{j} N(y ; H x, R) N(x ; \mu, \Sigma)}{\sum_{j=1}^{m} \sum_{i=1}^{n} \omega_{i} \beta_{j} \int N(y ; H x, R) N(x ; \mu, \Sigma) d x} \\
& =\frac{\sum_{j=1}^{m} \sum_{i=1}^{n} \omega_{i} \beta_{j} N(y ; c, D) N(x ; a, B)}{\sum_{j=1}^{m} \sum_{i=1}^{n} \omega_{i} \beta_{j} N(y ; c, D)}
\end{aligned}
$$

Note that:

$$
N(H x, R) N(x ; \mu, \Sigma)=\frac{1}{\sqrt{|R||\Sigma|}(\sqrt{2 \pi})^{n_{x}+n_{y}}} \exp \left(-\frac{Q}{2}\right)
$$

where:

$$
\begin{aligned}
& Q=(y-H x)^{T} R^{-1}(y-H x)+(x-\mu)^{T} \Sigma^{-1}(x-\mu) \\
&=x^{T} B^{-1} x-2 b x+y^{T} R^{-1} y+\mu^{T} \Sigma^{-1} \mu \\
&=(x-a)^{T} B^{-1}(x-a)-a^{T} B^{-1} a+y^{T} R^{-1} y+\mu^{T} \Sigma^{-1} \mu \\
& \approx(x-a)^{T} B^{-1}(x-a)+(y-c)^{T} D^{-1}(y-c) \\
& B^{-1}=H^{T} R^{-1} H+\Sigma^{-1} ; \quad b=y^{T} R^{-1} H+\mu^{T} \Sigma^{-1} \quad ; \quad a=B b \\
& D^{-1}=R^{-1}-R^{-1} H B^{-1} H^{T} R^{-1} \quad ; \quad d=\mu^{T} \Sigma^{-1} B^{-1} H^{T} R^{-1} \quad ; \quad c=D d
\end{aligned}
$$

so that:

$$
\begin{aligned}
N(x ; \mu, \Sigma) N(y ; H x, R) & =\frac{1}{\sqrt{|R||\Sigma|}(\sqrt{2 \pi})^{n_{x}+n_{y}}} \exp \left\{-\frac{1}{2}\left[(x-a)^{T} B^{-1}(x-a)+(y-c)^{T} D^{-1}(y-c)\right]\right\} \\
& =N(x ; a, B) N(y ; c, D)
\end{aligned}
$$


For a recursive algorithm, it is assumed that the probability density function of the initial condition $p\left(x_{0}\right)$ is approximated by:

$$
p\left(x_{0}\right) \approx \sum_{i=1}^{n} \omega_{i, 0} N\left(x_{0} ; \mu_{i, 0 \mid 0}, \Sigma_{i, 0 \mid 0}\right), \quad ; \quad \sum_{i=1}^{n} \omega_{i, 0}=1
$$

Without loss of generality, it is assumed that in an instant $k-1$ the system states are known $p\left(x_{k-1} \mid y_{1: k-1}\right)$, that is:

$$
p\left(x_{k-1} \mid y_{1: k-1}\right) \approx \sum_{i=1}^{n} \beta_{i, k-1} N\left(x_{k-1} ; \mu_{i, k-1 \mid k-1}, \Sigma_{i, k-1 \mid k-1}\right), \quad ; \quad \sum_{i=1}^{n} \beta_{i, k-1}=1
$$

If $x_{k}=M_{k}\left(x_{k-1}, u_{k}\right) \approx \sum_{i=1}^{n} \alpha_{i, k} N\left(x_{k} ; \mu_{i, k \mid k-1}, \Sigma_{i, k \mid k-1}\right)$, then it is assumed that $x_{k+1} \mid x_{k}$ is given by:

$$
p\left(x_{k+1} \mid x_{k}\right) \approx \sum_{i=1}^{n} \alpha_{i, k} N\left(x_{k+1} ; \mu, \Sigma\right) \quad ; \quad \sum_{i=1}^{n} \alpha_{i, k}=1
$$

where:

$$
\left.\mu=\sum_{i=1}^{n} \alpha_{i, k-1} \mu_{i, k \mid k-1} \quad ; \quad \Sigma=\sum_{i=1}^{n} \omega_{i, k}\left[\Sigma_{i, k \mid k-1}\right)+\left(\mu_{i, k \mid k-1}-\mu\right)\left(\mu_{i, k \mid k-1}-\mu\right)^{T}\right]
$$

Then the conditional state $x_{k}$, given the observed data $y_{1: k-1}$, is approximated by:

$$
\begin{aligned}
p\left(x_{k} \mid y_{1: k-1}\right) & =\int p\left(x_{k} \mid x_{k-1}\right) p\left(x_{k-1} \mid y_{1: k-1}\right) d x_{k-1} \\
& \approx \sum_{i=1}^{n} \sum_{j=1}^{m} \alpha_{j, k-1} \beta_{i, k-1} \mathbf{I}_{i, j}\left(x_{k}\right)
\end{aligned}
$$

where:

$$
\begin{gathered}
\mathbf{I}_{i, j}\left(x_{k}\right)=\int N\left(x_{k} ; \mu, \Sigma\right) N\left(x_{k-1} ; \mu_{i, k \mid k-1}, \Sigma_{i, k \mid k-1}\right) d x_{k-1} \\
=\int c_{i, k} N\left(x_{k} ; \mu_{i, k \mid k}^{1 b}, \Sigma_{i, k \mid k}^{1 b}\right) d x_{k-1} \\
\mu_{i, k \mid k-1}^{1 b}=\left(\Sigma^{-1}+\left(\Sigma_{i, k \mid k-1}\right)^{-1}\right)^{-1}\left(\Sigma^{-1} \mu+\left(\Sigma_{i, k \mid k-1}\right)^{-1} \mu_{i, k \mid k-1}\right) \\
\Sigma_{i, k \mid k-1}^{1 b}=\left(\Sigma^{-1}+\left(\Sigma_{i, k \mid k-1}\right)^{-1}\right)^{-1}
\end{gathered}
$$

and

$$
c_{i, k}=\frac{1}{(2 \pi)^{\frac{n+m}{2}}\left|\Sigma^{-1}+\left(\Sigma_{i, k \mid k-1}\right)^{-1}\right|^{\frac{1}{2}}} \exp -\frac{1}{2}\left[\left(\mu_{i, k \mid k-1}-\mu\right)^{T}\left(\Sigma^{-1}+\left(\Sigma_{i, k \mid k-1}\right)^{-1}\right)^{-1}\left(\mu_{i, k \mid k-1}-\mu\right)\right] .
$$

The assessment of $\mathbf{I}_{i, j}\left(x_{k}\right)$ can be treated as an estimation problem of a nonlinear process with non-Gaussian distribution. In the literature, they have used techniques such as Kalman filter assemblies, or Kalman filter sigma points, to approximate $\mathbf{I}_{i, j}\left(x_{k}\right)$ (see [41]). Furthermore, if it is assumed that the likelihood of the data is distributed according a Gaussian:

$$
p\left(y_{k} \mid x_{k}\right) \sim N\left(y_{k} ; H x_{k}, R_{k}\right)
$$


then, the probability density function of the filtered distribution (the probability distribution function modeling the approximate solutions) is approximated by:

$$
\begin{aligned}
p\left(x_{k} \mid y_{1: k}\right) & \propto p\left(y_{k} \mid x_{k}\right) p\left(x_{k} \mid y_{1: k-1}\right) \\
& =N\left(y_{k} ; H x_{k}, R_{k}\right) \sum_{i=1}^{n} \sum_{j=1}^{m} \alpha_{j, k-1} \beta_{i, k-1} \mathbf{I}_{i, j}\left(x_{k}\right) \\
& =\sum_{i=1}^{n} \sum_{j=1}^{m} \alpha_{j, k-1} \beta_{i, k-1} \int c_{i, k} N\left(y_{k} ; H x_{k}, R_{k}\right) N\left(x_{k} ; \mu_{i, k \mid k-1}^{1 b}, \Sigma_{i, k \mid k-1}^{1 b}\right) d x_{k-1} \\
& =\sum_{i=1}^{n} \sum_{j=1}^{m} \alpha_{j, k} \beta_{i, k-1} \int c_{i, k} c_{i, k}^{*} N\left(x_{k} ; \mu_{k \mid k}^{2 b}, \Sigma_{k \mid k}^{2 b}\right) d x_{k-1}
\end{aligned}
$$

where:

$$
\begin{gathered}
\mu_{i, k \mid k}^{2 b}=\left(R_{k}^{-1}+\left(\Sigma_{i, k \mid k-1}^{1 b}\right)^{-1}\right)^{-1}\left(R_{k}^{-1} H x_{k}+\left(\Sigma_{i, k \mid k-1}^{1 b}\right)^{-1} \mu_{i, k \mid k-1}^{1 b}\right) \\
\Sigma_{i, k \mid k}^{2 b}=\left(R_{k}^{-1}+\left(\Sigma_{i, k \mid k-1}^{1 b}\right)^{-1}\right)^{-1}
\end{gathered}
$$

and

$$
c_{i, k}^{*}=\frac{1}{(2 \pi)^{\frac{n+m}{2}}\left|R_{k}^{-1}+\left(\Sigma_{i, k \mid k-1}^{1 b}\right)^{-1}\right|^{\frac{1}{2}}} \exp -\frac{1}{2}\left[\left(\mu_{i, k \mid k-1}^{1 b}-H x_{k}\right)^{T}\left(R_{k}^{-1}+\left(\Sigma_{i, k \mid k-1}^{1 b}\right)^{-1}\right)^{-1}\left(\mu_{i, k \mid k-1}^{1 b}-H x_{k}\right)\right]
$$

A summary of the algorithm used to estimate the density function by GMF is shown in the following procedure:

\section{Algorithm 2: GMF}

- Step 1: Simulate the initial states $p\left(x_{0}\right)$ as shown in equation (39).

- Step 2: Use the Algorithm 1 to estimate the density of solutions.

- Step 3: Predict $p\left(x_{k} \mid y_{1: k-1}\right)$, using the equation(42).

- Step 4: Update $p\left(x_{k} \mid y_{1: k}\right)$, using the equation (44).

\subsection{Nonparametric filter particles (NPF)}

Suppose we want to approximate the filtered density $p\left(x_{k} \mid y_{1: k}\right)$ using an importance function in a marginal state space $x_{k}$, that is:

$$
\begin{aligned}
p\left(x_{k} \mid y_{1: k}\right) & \propto p\left(y_{k} \mid x_{k}\right) p\left(x_{k} \mid y_{1: k-1}\right) \\
& =p\left(y_{k} \mid x_{k}\right) \int p\left(x_{k} \mid x_{k-1}\right) p\left(x_{k-1} \mid y_{1: k-1}\right) d x_{k-1}
\end{aligned}
$$

A strategy for estimating the filtered distribution is to use a Sequential Monte Carlo method known as Particle filters. The particle filters consider $\left\{x_{k}^{(i)}, w_{k}^{(i)}\right\}_{i=1}^{n}$ a random sample which characterize the posterior probability density function $p\left(x_{k} \mid y_{1: k}\right)$. The weights are chosen such that $\sum_{i=1}^{n} W_{k}^{(i)}=1$. Then the posterior distribution, also called the filtered distribution in time $k$, can be approximated by an empirical distribution formed by the mass points or particles:

$$
p_{n}\left(x_{k} \mid y_{1: k}\right) \approx \sum_{i=1}^{n} w_{k}^{(i)} \delta\left(x_{k}-x_{k}^{(i)}\right)
$$


where $\delta($.$) is the Dirac delta function. Given the posterior distribution, some quantities of interest can be estimated,$ such as the expected values of a function $g\left(x_{k}\right)$ associated with the filtered distribution $p\left(x_{k} \mid y_{1: k}\right)$, that is:

$$
E\left[g\left(x_{k}\right)\right]=\int g\left(x_{k}\right) p\left(x_{k} \mid y_{1: k}\right) d x_{k}
$$

Some of the researchers who worked on the subject are: [71]; [20]; [32]; [37]; [11]; [38]; [55]; [10]; [17]; [19]; [39] among others.

The weights $w_{k}^{(i)}$ are chosen using the principle of importance sampling. The principle of importance sampling is based on the following argument: Suppose that $p(x) \propto \gamma(x)$, is a probability density which is difficult to sample, but $\gamma(x)$ can be evaluated and consequently $p(x)$ also be evaluated to a proportionality constant. Then proceed as follows: Let $x^{(i)} \sim q(x), i=1, \ldots, n$ be a generated sample of a proposed distribution $q($.$) , called density of$ importance. Then a weighted density approximation of $p($.$) is given by:$

$$
p(x) \approx \sum_{i=1}^{n} w^{(i)} \delta\left(x-x^{(i)}\right)
$$

where:

$$
w^{(i)} \propto \frac{\gamma\left(x^{(i)}\right)}{q\left(x^{(i)}\right)}
$$

The $w^{(i)}$ represents a standardized weight of the $i$-th particle. If samples $\left\{x_{0: k}^{(i)}\right\}$ are taken using a density of importance $q\left(x_{k} \mid y_{1: k}\right)$ then the weights used to approximate the equation (46) are obtained by:

$$
w_{k}^{(i)} \propto \frac{p\left(x_{k}^{(i)} \mid y_{1: k}\right)}{q\left(x_{k}^{(i)} \mid y_{1: k}\right)}
$$

If the density of importance can be factored so that:

$$
q\left(x_{k} \mid y_{1: k}\right)=q\left(x_{k} \mid x_{k-1}, y_{1: k}\right) q\left(x_{k-1} \mid y_{1: k-1}\right)
$$

then we can get the samples $x_{0: k}^{(i)}$ from $q\left(x_{k} \mid y_{1: k}\right)$ by increasing each of the samples $x_{0: k-1}^{(i)}$ that already exist and are obtained from $q\left(x_{k-1}^{(i)} \mid y_{1: k-1}\right)$, generating the new state $x_{k}^{(i)}$ of $q\left(x_{k} \mid x_{k-1}, y_{1: k}\right)$. For the updated weights, the filtered distribution $p\left(x_{k} \mid y_{1: k}\right)$ is expressed in terms of the equation given in (45). Updated weights are given by:

$$
\begin{aligned}
w_{k}^{(i)} & \propto \frac{p\left(y_{k} \mid x_{k}^{(i)}\right) p\left(x_{k}^{(i)} \mid x_{k-1}^{(i)}\right) p\left(x_{k-1}^{(i)} \mid y_{1: k-1}\right)}{q\left(x_{k}^{(i)} \mid x_{k-1}^{(i)}, y_{1: t}\right) q\left(x_{k-1}^{(i)} \mid y_{1: k-1}\right)} \\
& =\frac{p\left(y_{k} \mid x_{k}^{(i)}\right) p\left(x_{k}^{(i)} \mid x_{k-1}^{(i)}\right)}{q\left(x_{k}^{(i)} \mid x_{k-1}^{(i)}, y_{1: k}\right)} w_{k-1}^{(i)}
\end{aligned}
$$

where

$$
w_{k-1}^{(i)}=\frac{p\left(x_{k-1}^{(i)} \mid y_{1: k-1}\right)}{q\left(x_{k-1}^{(i)} \mid y_{1: k-1}\right)}
$$

In particular, if $q\left(x_{k} \mid x_{k-1}, y_{1: k}\right)=q\left(x_{k} \mid x_{k-1}, y_{k}\right)$, then the density of importance depends only on $x_{k-1}$, and $y_{k}$. This situation is appropriate when required to obtain the filtered estimator $p\left(x_{k} \mid y_{1: k}\right)$ in real time $k$. Then the weights are modified as follows:

$$
w_{k}^{(i)}=\frac{p\left(y_{k} \mid x_{k}^{(i)}\right) p\left(x_{k}^{(i)} \mid x_{k-1}^{(i)}\right)}{q\left(x_{k}^{(i)} \mid x_{k-1}^{(i)}, y_{k}\right)} w_{k-1}^{(i)}
$$


The filtered density $p_{n}\left(x_{k} \mid y_{1: k}\right)$ can be approximated by:

$$
p_{n}\left(x_{k} \mid y_{1: k}\right) \approx \sum_{i=1}^{n} w_{k}^{(i)} \delta\left(x_{k}-x_{k}^{(i)}\right)
$$

It was proved in [12] that when $n \rightarrow \infty$ the equation given in (52) approaches the true posterior distribution $p\left(x_{k} \mid y_{1: k}\right)$.

This paper seeks to approximate the filtered density function by using nonparametric statistical techniques. A Dirichlet mixing process is used to estimate the unknown density of states. The algorithm predicts the equation given in (12) and updates the equation given in (14) based on simulated samples. In order to implement the algorithm, it is supposed to start with a set of random samples $\left\{x_{0: k-1}^{(i)}\right\}_{i=0}^{n}$ generated by a known distribution function $p\left(x_{k-1} \mid y_{1: k-1}\right)$. The procedure is summarized as follows:

\section{Algorithm 3: NPF}

- Step 1: Initialize with a nonparametric prior distribution of an initial state: (for $k=0$, and $i=1, \ldots, n)$ :

$$
\tilde{x}_{0}^{(i)} \sim p\left(x_{0}\right) \quad ; \quad w_{0}^{(i)}=1
$$

where the samples $\left\{x_{0: k-1}^{(i)}\right\}$ are considered known from the posterior distribution $p\left(x_{k-1} \mid y_{1: k-1}\right)$.

- Step 2: Perform the nonparametric importance sampling: (for $k \geq 1$ )

- For $i=1, \ldots, n$ :

$$
\tilde{x}_{k}^{(i)} \sim p\left(x_{k} \mid \tilde{x}_{k-1}^{(i)}\right)
$$

- Step 3: Obtain an estimated prediction density:

$$
p_{n}\left(x_{k} \mid y_{1: k-1}\right)=\sum_{i=1}^{n} w_{k-1}^{(i)} \delta\left(x_{k}^{(i)}-\tilde{x}_{k}^{(i)}\right)
$$

using samples $\left\{\tilde{x}_{k}^{(i)}, w_{k-1}^{(i)}\right\}_{i=1}^{n}$. For example, the predictive mean and covariance can be estimated as follows:

$$
\begin{gathered}
m_{k \mid k-1}=\sum_{i=1}^{n} w_{k-1}^{(i)} \tilde{x}_{k}^{(i)} \\
P_{k \mid k-1}=\sum_{i=1}^{n} w_{k-1}^{(i)}\left(\tilde{x}_{k}^{(i)}-m_{k \mid k-1}\right)\left(\tilde{x}_{k}^{(i)}-m_{k \mid k-1}\right)^{T}
\end{gathered}
$$

- Step 4: For $i=1, \ldots, n$, the importance density is sampled:

$$
\tilde{x}_{k}^{(i)} \sim q\left(x_{k} \mid y_{1: k}\right)
$$

and the importance weights are calculated:

$$
\tilde{w}_{k}^{(i)} \propto \frac{p\left(y_{k} \mid \tilde{x}_{k}^{(i)}\right) p_{n}\left(\tilde{x}_{k}^{(i)} \mid y_{1: k-1}\right)}{q\left(\tilde{x}_{k}^{(i)} \mid y_{1: k}\right)}
$$

- Step 5: Density prediction $p_{n}\left(x_{k} \mid y_{1: k}\right)$ is updated using the sample $\left\{\tilde{x}_{k}^{(i)}, \tilde{w}_{k}^{(i)}\right\}_{i=1}^{n}$. 
- Step 6: For $i=1, \ldots, n$, sample:

$$
\tilde{x}_{k}^{(i)} \sim p_{n}\left(x_{k} \mid y_{1: k}\right)
$$

and calculate the importance weights:

$$
\tilde{w}_{k}^{(i)} \propto \frac{p\left(y_{k} \mid \tilde{x}_{k}^{(i)}\right) p_{n}\left(\tilde{x}_{k}^{(i)} \mid y_{1: k-1}\right)}{p_{n}\left(\tilde{x}_{k}^{(i)} \mid y_{1: k}\right)}
$$

- Step 7: NPF outputs consist of particles $\left\{\tilde{x}_{k}^{(i)}, \tilde{w}_{k}^{(i)}\right\}_{i=1}^{n}$, which approximate the filtered density function:

$$
p\left(x_{k}, k\right)=p_{n}\left(x_{k} \mid y_{1: k}\right)=\sum_{i=1}^{n} \tilde{w}_{k}^{(i)} \delta\left(x_{k}^{(i)}-\tilde{x}_{k}^{(i)}\right)
$$

The updated filtered distribution can be characterized by the mean and covariance:

$$
\begin{gathered}
m_{k \mid k}=\sum_{i=1}^{n} \tilde{w}_{k}^{(i)} \tilde{x}_{k}^{(i)} \\
P_{k \mid k}=\sum_{i=1}^{n} \tilde{w}_{k}^{(i)}\left(\tilde{x}_{k}^{(i)}-m_{k \mid k}\right)\left(\tilde{x}_{k}^{(i)}-m_{k \mid k}\right)^{T}
\end{gathered}
$$

\subsection{Gaussian particle filter (GPF)}

The interest in the following line is to present a method for approximating the solution using a Gaussian Particle Filter (GPF) as proposed in [34]. This algorithm allows the generation of samples that approximate the predictive distribution $p\left(x_{k} \mid y_{1: k-1}\right)$, and the filtered distribution $p\left(x_{k} \mid y_{1: k}\right)$ using Gaussian densities, where the vector of means and the variance-covariance matrix are estimates of the particles generated. If at time $k-1$, the solution is approximated by $N\left(\mu_{k-1}, \Sigma_{k-1}\right)$, and at time $k$, it is approximated by $N\left(\tilde{\mu}_{k}, \tilde{\Sigma}_{k}\right)$, then the steps to implement the $G P F$ consist of the following:

\section{Algorithm 4: GPF}

- Step 1: Generate $x_{k-1}^{(m)} \mid \mu_{k-1}, \Sigma_{k-1} \sim N\left(\mu_{k-1}, \Sigma_{k-1}\right)$.

- Step 2: Generate $\mu_{k} \mid \Sigma_{k-1}, \lambda, \tau \sim N\left(\lambda, \frac{\Sigma_{k-1}}{\tau}\right)$.

- Step 3: Generate $\Sigma_{k} \mid \Sigma_{0}, \nu \sim I W\left(\Sigma_{0}, \nu\right)$, and do:

$$
x_{k}^{(m)}=M_{k}\left(x_{k-1}^{(m)}, u_{k}^{(m)}\right)=\sum_{k=1}^{n} \omega_{k} N\left(\mu_{k}, \Sigma_{k}\right) \quad ; \quad \sum_{k=1}^{n} \omega_{k}=1
$$

- Step 4: Generate $x_{k+1}^{(m)} \mid x_{k}^{(m)} \sim N(\mu, \Sigma)$.

- Step 5: Generate $\tilde{w}_{k}^{(m)}=p\left(y_{k} \mid x_{k+1}^{(m)}\right) \sim N\left(H x_{k+1}^{(m)}, R_{t}\right)$.

- Step 6: The weights are normalized $w_{k}^{(m)}=\frac{\tilde{w}_{t}^{m}}{\sum_{k=1}^{t} \tilde{w}_{k}^{m}}$.

- Step 7: Estimate $\tilde{\mu}_{k}$, and $\tilde{\Sigma}_{k}$ by:

$$
\begin{gathered}
\tilde{\mu}_{k}=\sum_{m=1}^{M} w_{k}^{(m)} x_{k+1}^{(m)} \\
\tilde{\Sigma}_{k}=\sum_{m=1}^{M} w_{k}^{(m)}\left(x_{k+1}^{(m)}-\tilde{\mu}_{k}\right)\left(x_{k+1}^{(m)}-\tilde{\mu}_{k}\right)^{T}
\end{gathered}
$$

- Step 8: Approximate $p\left(x_{k}, k\right)=p\left(x_{k} \mid y_{1: k}\right) \sim N\left(\tilde{\mu}_{k}, \tilde{\Sigma}_{k}\right)$. 


\section{Results}

The first instance consists of a linear case, while the second example consists of a case with a nonlinear structure. To measure the estimation quality of the proposed algorithms we use the square root of the mean square error (RMSE) defined as:

$$
R M S E=\frac{1}{n} \sum_{i=1}^{n}\left\|\hat{x}_{k}^{(i)}-m_{k \mid k}\right\|_{2}
$$

where $\hat{x}_{k}^{(i)}$ is an estimator of $\mathbb{E}\left(x_{k} \mid y_{1: k}\right)$, and $m_{k \mid k}=\sum_{i=1}^{n} w_{k}^{(i)} x_{k}^{(i)}$.

\subsection{Example 1}

As a first example, the Lorenz model is implemented ([40]), representing a coupled system of nonlinear differential equations, given by:

$$
\begin{aligned}
& \frac{d x}{d t}=s(y-x) \\
& \frac{d y}{d t}=r x-y-x z \\
& \frac{d z}{d t}=x y-b z
\end{aligned}
$$

where: $s, r, b$ are parameters. The state vector $\mathbf{x}=(x, y, z)^{T}$ represents a position of the particles in the phase space. The model is discretized using the first order Euler method $x_{t}=x_{t-1}+h f\left(x_{t-1}\right)$, with step size $h$. The discrete evolution equation is given by:

$$
\begin{aligned}
& x_{t+1}=x_{t}+h\left(s\left(y_{t}-x_{t}\right)\right) \\
& y_{t+1}=y_{t}+h\left(r x_{t}-y_{t}-x_{t} z_{t}\right) \\
& z_{t+1}=z_{t}+h\left(x_{t} y_{t}-b z_{t}\right)
\end{aligned}
$$

where: $s=10, r=28, \mathrm{y} b=\frac{8}{3}$. The data was generated by a linear observation equation as proposed in [9]:

$$
\gamma_{t}=\phi_{t}+\eta_{t}
$$

where: $\gamma_{t}=\left(x_{t}^{+}, y_{t}^{+}, z_{t}^{+}\right)^{T}, \phi_{t}=\left(x_{t}, y_{t}, z_{t}\right)^{T}$, and $\eta_{t}=\left(\eta_{x_{t}}, \eta_{y_{t}}, \eta_{x_{t}}\right)^{T}, \eta_{t} \sim N\left(\mathbf{0}, \sigma_{\eta}^{2} \mathbf{I}\right), \mathbf{0}$ is a vector of zeros, and $\mathbf{I}$ is the identity matrix.

To initialize the algorithm the following a priori distributions are considered:

- For GMF: $h=0.0018, \mu_{x_{0 \mid 0}}=0.2294, \sigma_{x_{0 \mid 0}}=0.001, \mu_{y_{0 \mid 0}}=1.636, \sigma_{y_{0 \mid 0}}=0.001, \mu_{z_{0 \mid 0}}=20.81, \sigma_{z_{0 \mid 0}}=$ 0.001, wishardf $f_{x}=10$, wishardf $_{y}=100$ and wishardf $_{z}=10$.

- For NPF: $h=0.0018, \mu_{x_{0 \mid 0}}=0.2294, \sigma_{x_{0 \mid 0}}=0.001, \mu_{y_{0 \mid 0}}=1.636, \sigma_{y_{0 \mid 0}}=0.001, \mu_{z_{0 \mid 0}}=20.81, \sigma_{z_{0 \mid 0}}=$ $0.001, \sigma_{\eta_{x}}=0.01, \sigma_{\eta_{y}}=0.01, \sigma_{\eta_{z}}=0.01$, wishardf $_{x}=10$, wishardf $_{y}=100$ and wishardf $_{z}=10$.

- For GPF: $h=0.0018, \mu_{x_{0 \mid 0}}=0.2294, \sigma_{x_{0 \mid 0}}=0.001, \mu_{y_{0 \mid 0}}=1.636, \sigma_{y_{0 \mid 0}}=0.001, \mu_{z_{0 \mid 0}}=20.81, \sigma_{z_{0 \mid 0}}=$ $0.001, \sigma_{\eta_{x}}=0.01, \sigma_{\eta_{y}}=0.01, \sigma_{\eta_{z}}=0.01$, wishardf $_{x}=10$, wishardf $_{y}=10$ and wishardf $_{z}=10$.

In Table I, the results of the goodness of fit measure, used to gauge the estimation quality of the algorithms, are shown. In this case, the RMSE calculations in the table shows no significant differences in the estimated errors and the computing time. Figure 1, shows three graphs depicting the reconstruction of the true Lorenz attractor and the posterior means of the estimated solution by the three algorithms proposed, where it can be seen that all filters fit almost perfectly to the original map of Lorenz. 


\begin{tabular}{|l|r|r|r|}
\hline RMSE & GMF & NPF & GPF \\
\hline State $x$ & 0.0235 & 0.0458 & 0.0328 \\
State $y$ & 2.2599 & 2.2421 & 2.2602 \\
State $z$ & 0.0228 & 0.0237 & 0.0108 \\
Time (secs.) & 2.1326 & 2.4443 & 1.9424 \\
\hline
\end{tabular}

Table I. RMSE and computing time of the algorithms GMF, NPF, and GPF.
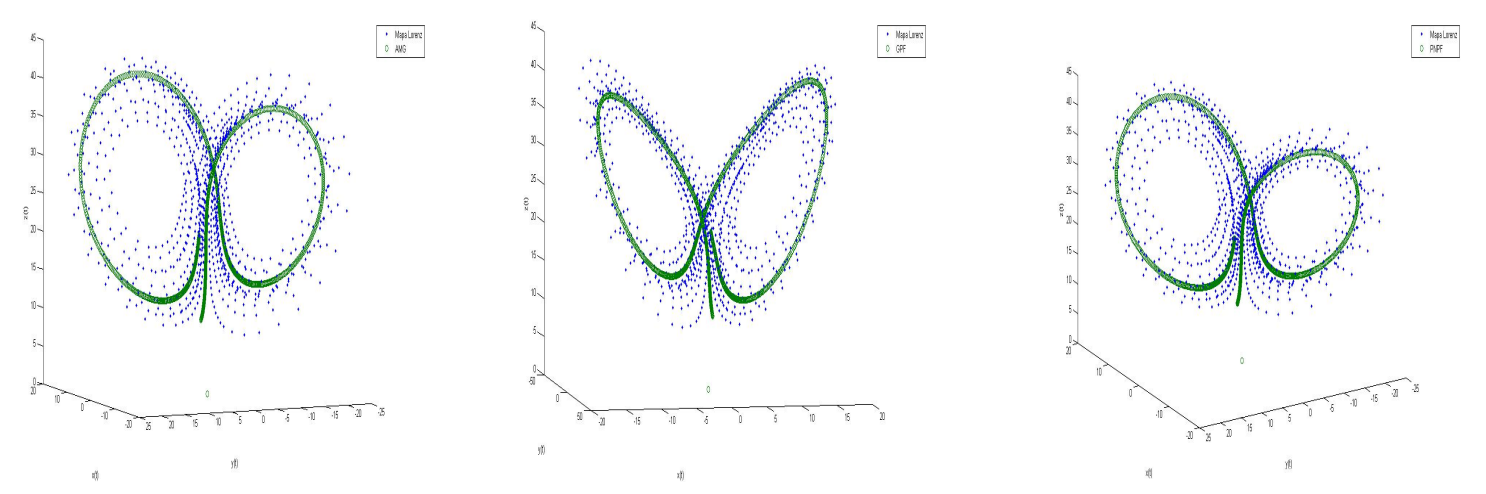

Figure 1. Lorenz attractor reconstruction using the filters GMF, NPF and GPF.

\subsection{Example 2}

The second example is a temporary space model based on a stochastic differential equation for predicting rainfall described in [4], where $x_{t}$ denotes the total rainfall at time $t$ in a locality of $\mathbf{s}=(x, y) \in \mathbf{R}^{2}$; and $x_{0}$ is an initial state. Suppose that the change in total rainfall during a very short time interval $\Delta t$ and $(\Delta x)_{1}=\gamma$, and $(\Delta x)_{2}=0$ have probabilities $p_{1}=\lambda \Delta t$, and $p_{2}=1-\lambda \Delta t$, respectively. The expected value and the mean squared change in the total precipitation is given by:

$$
\begin{aligned}
& E(\Delta x)=\gamma \lambda \Delta t \\
& E\left((\Delta x)^{2}\right)=\gamma^{2} \lambda \Delta t
\end{aligned}
$$

Letting $\mu=\gamma \lambda \Delta t$, and $\sigma=\sqrt{\gamma^{2} \lambda} \Delta t$, a stochastic differential equation to model the total precipitation $x_{t}$ is obtained:

$$
\begin{aligned}
& d \mathbf{x}_{t}=\mu d t+\sigma d \mathbf{W}_{t} \\
& x_{0}=0
\end{aligned}
$$

where $d \mathbf{W}_{t}$ is a vector of a Brownian process with mean $\mathbf{0}$ and covariance matrix $\mathbf{Q}_{t}$. The evolution in time of the marginal density of the state is given by the posterior marginal distribution $p\left(x_{k} \mid y_{1: k-1}\right)$, which satisfies the Fokker-Planck equation:

$$
\frac{\partial}{\partial t} p\left(x_{k} \mid y_{1: k-1}\right)=\mathcal{L}\left(p\left(x_{k} \mid y_{1: k-1}\right)\right.
$$

where $\mathcal{L}$ is a diffusion operator defined by:

$$
\mathcal{L}(p)=-\sum_{i=0}^{n_{p}} \frac{\partial(\mu p)}{\partial x_{i}}+\frac{1}{2} \sum_{i=0}^{n_{p}} \sum_{j=0}^{n_{p}} \frac{\partial^{2}\left[\left(\sigma Q_{t} \sigma^{T}\right)_{i j} p\right]}{\partial x_{i} x_{j}}
$$


with $p=p\left(x_{k} \mid y_{1: k-1}\right)$. The initial condition is given by $p\left(x_{k-1} \mid y_{1: k-1}\right)$. Applying the Fokker-Planck equation to the equation (58), we obtain:

$$
\frac{\partial p}{\partial t}=-\mu \frac{\partial p}{\partial x}+\frac{\sigma^{2} Q_{t}}{2} \frac{\partial^{2} p}{\partial x^{2}}
$$

The next step is to find a solution to the equation given in (61). The method of discretization of Crank and Nicolson was used to do this. See [59] for detailed. To illustrate the methodology, a data series of daily precipitation was considered. The data included information from January 2011 until May 2012 for three (3) meteorological stations located in Arágua (Ceniap, Tamarindo and Tucutunemo), Venezuela. The data are available in http: //agrometeorologia.inia.gob.ve/.

To initialize the algorithm GMF, the following a priori distributions were considered:

- Ceniap station $\delta_{t}=0.001, \delta_{x}=0.001, \sigma=100, Q_{t}=0.0001$, wishardf $=10, \mu=18.5, u=100, v=100$.

- Tucutunemo station $\delta_{t}=0.1, \delta_{x}=0.1, \sigma=10, Q_{t}=0.0001$, wishardf $=10, \mu=18.5, u=10, v=10$.

- Tamarindo station $\delta_{t}=0.001, \delta_{x}=0.001, \sigma=10, Q_{t}=0.0001$, wishardf $=10, \mu=18.5, u=100, v=$ 100.

To initialize the algorithm NPF, the following prior distributions were considered:

- Ceniap station delta $a_{t}=0.001$, delta $a_{x}=0.001$, sigma $=100$, miu $=18.56, q_{t}=0.0001$, wishardf $=10$, $u=100$ and $v=100$.

- Tamarindo station delta $a_{t}=0.001$, delta $a_{x}=0.001$, sigma $=100$, miu $=18.56, q_{t}=0.0001$, wishardf $=$ $10, u=100$ and $v=100$.

- Tucutunemo station delta $a_{t}=0.1$, delta $a_{x}=0.1$, sigma $=100$, miu $=18.56, q_{t}=0.0001$, wishardf $=10$, $u=10$ and $v=10$.

To initialize the algorithm GPF, the following prior distributions were considered:

- Ceniap station delta $a_{t}=0.001$, delta $a_{x}=0.001$, sigma $=10$, miu $=18.56, q_{t}=0.0001$, wishardf $=10$, $u=100$ and $v=100$.

- Tamarindo station delta $a_{t}=0.1$, delta $a_{x}=0.1$, sigma $=10$, miu $=18.56, q_{t}=0.0001$, wishardf $=10$, $u=100$ and $v=100$.

- Tucutunemo station delta $a_{t}=0.1$, delta $a_{x}=0.1$, sigma $=10$, miu $=18.56, q_{t}=0.0001$, wishardf $=10$, $u=10$ and $v=10$.

In table II, the RMSE estimated by the proposed algorithms are shown, where we can observe a low variability in the RMSE for the three filters in the Ceniap station. In the Tamarindo station, the lowest RMSE was obtained by the NPF, and the best estimated values in the Tucutunemo station were calculated with the GPF. The computing time for the three filters in all meteorological stations are similar, although the computing time of the NPF is slightly lower than the other two filters. In Figures 2, 3 and 4, the three graphs represent the real states in black, the simulated states using the GMF in red, the NPF in blue and the GPF in green for the three stations. We can see a very similar behavior between the actual values, and simulated values of the states by the three proposed algorithms.

\begin{tabular}{|l|r|r|r|}
\hline RMSE & GMF & NPF & GPF \\
\hline Ceniap & 0.0018 & 0.0082 & 0.0019 \\
Tamarindo & 0.1792 & 0.0790 & 0.1654 \\
Tucutunemo & 0.0540 & 0.0253 & 0.0074 \\
Time (secs.) & 0.4233 & 0.3912 & 0.4826 \\
\hline
\end{tabular}

Table II. RMSE and computing time of the algorithms GMF, NPF, and GPF. 


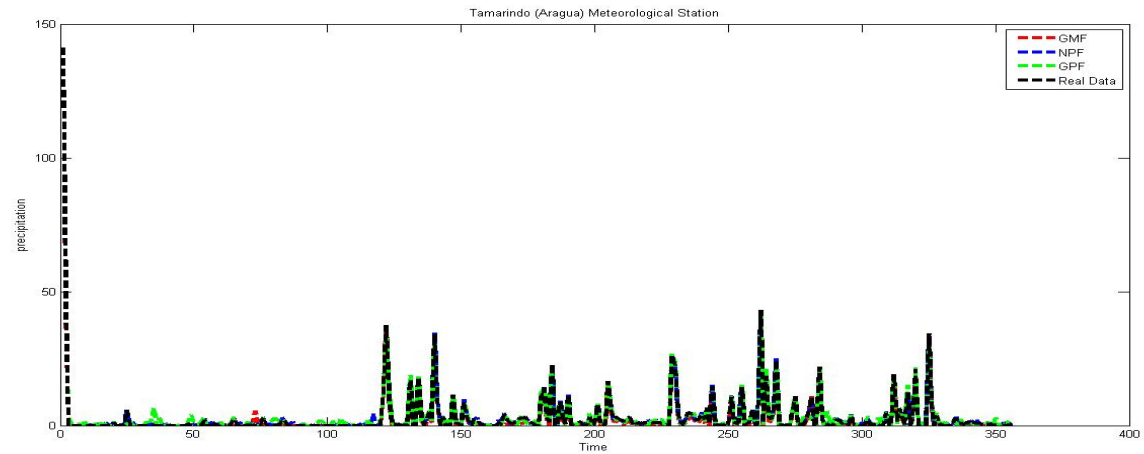

Figure 2. Real and estimated state by the GMF, NPF and GPF for Tamarindo station.

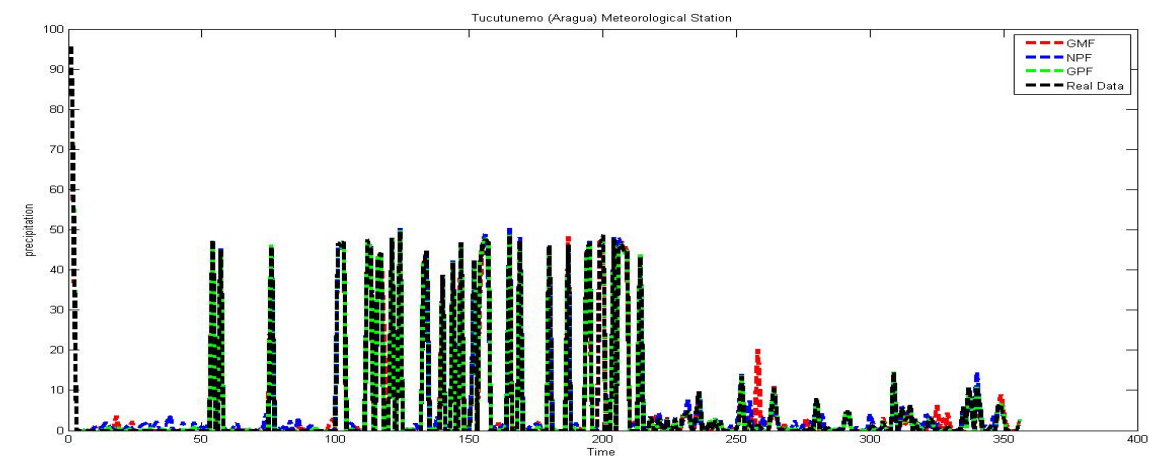

Figure 3. Real and estimated state by the GMF, NPF and GPF for Tucutunemo station.

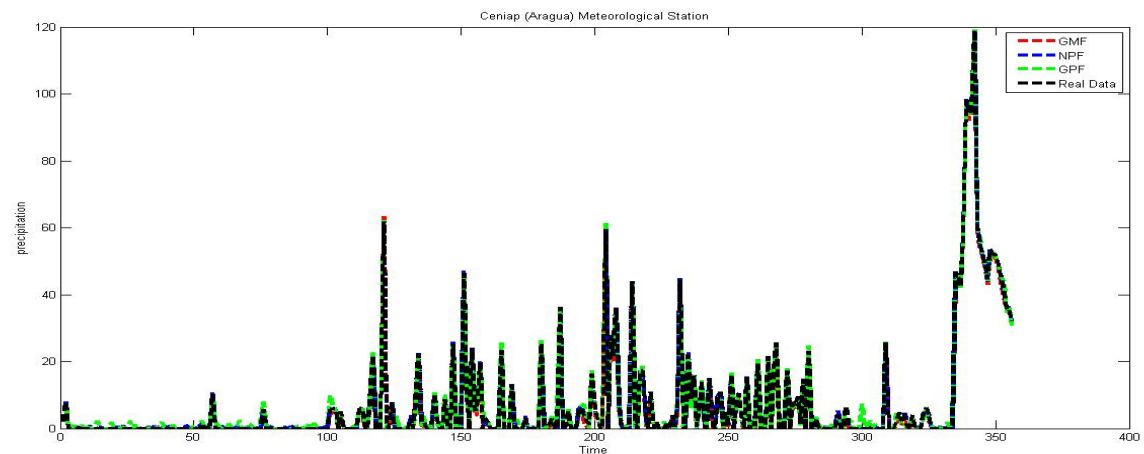

Figure 4. Real and estimated state by the GMF, NPF and GPF for Ceniap station. 


\section{Conclusions and discussion}

This article has introduced a Bayesian nonparametric estimation method to approximate the solutions of a general stochastic differential equation. The main contribution of the work is presenting an approach to combine mixtures of Dirichlet processes and mixtures of Gaussian processes in terms of state space models. The proposed hierarchical structures are extremely flexible with regard to the specifications of the prior assumptions of parameters, are easy to interpret and allow to model a variety of physical phenomena under uncertainty. In addition, we propose an efficient way to predict and update the states of the filtered distribution of the dynamic system through the GMF, NPF and GPF algorithms. The methodology was illustrated by means of two stochastic differential equations, one of them synthetic, and the other obtained from a real process. Since the approximate solutions were obtained from complex models, it is shown that the proposed filters have good performance in the reconstruction of the Lorenz attractor and the states of rainfall model. The computational implementation is also of low cost. It is also shown that the algorithms work well in the context of nonlinear dynamic processes, which require joint estimation of states and distributions of the noise of the equation of state. To measure the quality estimation of the algorithms the square root mean square error was used as a measure of goodness of fit. This measures produced insignificant errors of the estimation.

\section{REFERENCES}

1. Y. Ait-Sahalia, Maximum likelihood estimation of discretely sampled diffusions: a closed form approximation approach, Econometrica, vol. 70, no. 1, pp. 223-262, 2002.

2. B. Anderson, and J. Moore, Optimal Filtering, Prentice-Hall Information and system sciences series, Englewood Cliffs, New Jersey, 1979.

3. D. Alspach, and H. Sorenson, Nonlinear Bayesian Estimation Using Gaussian Sum Approximations, IEEE transactions on automatic control, vol. 17, no 4, pp. 439-448, 1972.

4. E. Allen, Modeling with Itô Stochastic Differential Equations, Springer, Texas Tech University, USA, 2007.

5. C. Archambeau, D. Cornford, M. Opper, J. Shawe-Taylor, Gaussian Process Approximations of Stochastic Differential Equations, JMLR. Workshop and Conference Proceedings, vol. 1, pp. 1-16, 2007.

6. S. Arulampalam, S. Maskell, N. Gordon and T. Clapp, A Tutorial on Particle Filters for On-line Non-linear/Non-Gaussian Bayesian Tracking, IEEE Transactions on signal processing, vol. 50, no. 2, pp. 174-188, 2002.

7. D. Blackwell, and J. MacQueen, Ferguson distributions via Polya urn schemes, The annals of statistics, pp. 353-355, 1973.

8. O. Calin, An Introduction to Stochastic Calculus with Applications to Finance, Ann Arbor, 2012.

9. C. Chui, G. Chen, Kalman Filtering with Real-Time Applications, Springer Science \& Business Media, 2008.

10. A. Doucet, J. De Freitas and N. Gordon, An introduction to sequential Monte Carlo methods, Sequential Monte Carlo methods in practice. Springer New York, pp. 3-14, 2001.

11. A. Doucet, S. Godsill and C. Andrieu, On sequential Monte Carlo sampling methods for Bayesian filtering, Statistics and computing, vol. 10, no. 3, pp. 197-208, 2000.

12. Dan Crisan and Arnaud Doucet, A Survey of Convergence Results on Particle Filtering Methods for Practitioners, IEEE Transactions on signal processing, vol. 50, no. 3, pp. 736-746, 2002.

13. O. Elerian, S. Chib, N. Shephard, Likelihood inference for discretely observed non-linear diffusions, Econometrica, vol. 69, no. 4, pp. 959-993, 2001.

14. L. Evans, An Introduction to Stochastic Differential Equations, American Mathematical Soc., 2012.

15. M. Escobar, and H. West, Bayesian density estimation and inference using mixtures, Journal of the American Statistical Association, vol. 90, no. 430, pp. 577-588, 1994.

16. T. Ferguson, A Bayesian analysis of some nonparametric problems, The annals of statistics, vol. 1, pp. 209-230, 1973.

17. W. Fong, S. Godsill, A. Doucet, M. West, Monte Carlo smoothing with application to audio signal enhancement, IEEE Transactions on Signal Processing, vol. 50, no. 2, pp. 438- 449, 2002.

18. A. Gelfand, A. Kottas, and S. MacEachern, Bayesian nonparametric spatial modeling with Dirichlet process mixing, Journal of the American Statistical Association, vol. 100, no. 471, pp. 1021-1035, 2005.

19. S. Godsill, A. Doucet and M. West, Monte Carlo smoothing for nonlinear Time series, Journal of the American statistical Association, 2012.

20. N. Gordon, D. Salmond and A. Smith, Novel approach to nonlinear/non-Gaussian Bayesian state estimation, IEE Proceedings F-Radar and Signal Processing. IET, pp. 107-113, 1993.

21. A. Guidoum, and K. Boukhetala, Quasi Maximum Likelihood Estimation for One Dimensional Diffusion Processes, In submission (The R Journal), 2014.

22. A. Hurn, K. Lindsay and V. Martin, On the efficacy of simulated maximum likelihood for estimating the parameters of stochastic differential equations, Journal of Time Series Analysis, vol. 24, no. 1, pp. 45-63, 2003.

23. A. Hurn, K. Lindsay, and A. McClelland, A quasi-maximum likelihood method for estimating the parameters of multivariate diffusions, Journal of Econometric, vol. 172, no. 1, pp. 106-126, 2013. 
24. K. Ito, and K. Xiong, Gaussian Filters for Nonlinear Filtering Problems, IEEE Transactions on Automatic Control, vol. 45, no. 5, pp. 910-927, 2000.

25. A. Jazwinski, Stochastic Processes and Filtering Theory, Academic Press, Inc. New York, USA, 1970.

26. B. Jensen, and R. Poulsen, Transition Densities of Diffusion Processes: Numerical Comparison of Approximation Techniques, Journal of Derivatives, vol. 9, no. 4, pp. 18-32, 2002.

27. C. S. Jones, A simple Bayesian method for the analysis of diffusion processes, Available at SSRN 111488, 1998.

28. S. Julier and J. Uhlmann, A new extension of the Kalman filter to nonlinear systems, AeroSense'97. International Society for Optics and Photonics, pp. 182-193, 1997.

29. S. Julier and J. Uhlmann, Unscented Filtering and Nonlinear Estimation, Proceedings of the IEEE, vol. 92, no. 3, pp. 401-422, 2004.

30. S. Julier, J. Uhlmann, and H. Durrant-White, A New Method for the Nonlinear Transformation of Means and Covariances in Filters and Estimators, IEEE Transactions on Automatic Control, vol. 45, pp. 477-478, 2000.

31. E. Kalnay, Atmospheric modeling, data assimilation and predictability, Cambridge University Press, 2003.

32. G. Kitagawa, Monte Carlo Filter and Smoother for Nonlinear Non Gaussian State Models, Journal of computational and graphical statistics, vol. 5, no. 1, pp. 1-25, 1996.

33. K. Kokkala, A. Solin, S. Sarkka, Sigma-Point Filtering and Smoothing Based Parameter Estimation in Nonlinear Dynamic Systems, arXiv preprint arXiv:1504.06173, 2015.

34. J. Kotecha, and P. Djuric, Gaussian sum particle filtering, IEEE Transactions on signal processing, vol. 51, no. 10, pp. 2602-2612, 2003.

35. P. Kloeden, and E. Platen, Numerical Solution of Stochastic Differential Equations, Springer Verlag, 1995.

36. P. Kloeden and E. Platen, Numerical Solution of Stochastic Differential Equations, Springer-Verlag, 1992.

37. J. Liu and R. Chen, Sequential Monte Carlo Methods for Dynamical Systems, Journal of the American statistical association, vol. 93, no. 443, pp. 1032-1044, 1998.

38. J. Liu and M. West, Combine Parameter and State Estimation in Simulation Based Filtering, Sequential Monte Carlo methods in practice. Springer New York, pp. 197-223, 2001.

39. M. Lin, J. Zhang, Q. Cheng, and R. Chen, Independent Particle Filters, Journal of the American Statistical Association, vol. 100, no. 472, pp. 1412-1421, 2005.

40. E. Lorenz, Deterministic Nonperiodic Flow, Journal of the atmospheric sciences, vol. 20, no. 2, pp. 130-141, 1963.

41. M. Luo, State estimation in high dimensional systems: the method of the ensemble unscented Kalman filter, Inference and Estimation in Probabilistic Time-Series Models, 2008.

42. S. MacEachem, and P. Muller, Estimating Mixture of Dirichlet Process Models, Journal of Computational and Graphical Statistics, vol. 7, no. 2 pp. 223-238, 1998.

43. P. Maybeck, Stochastic models, estimation, and control, Academic Press, vol. 3, 1982.

44. I. Mbalawata, S. Sarkka, H. Haario, Parameter estimation in stochastic differential equations with Markov chain Monte Carlo and nonlinear Kalman filtering, Computational Statistics, vol. 28, no. 3, pp. 1195-1223, 2013.

45. P. Muller, F. Quintana, Nonparametric Bayesian data analysis, Statistical science, pp. 95-110, 2004.

46. R. Neal, Markov chain sampling methods for Dirichlet process mixture models, Journal of Computational and Graphical Statistics, vol. 9, no. 2, pp. 249-265, 2000.

47. J. Neddermeyer, Sequential Monte Carlo Methods for General State-Space Models, Tesis Doctoral. Diplomarbeit (Master thesis), University of Heidelberg, 2007.

48. J. Neddermeyer, Computationally Efficient Nonparametric Importance Sampling, Journal of the American Statistical Association, vol. 104, no. 486 788-802, 2009.

49. J. Nicolau, New Technique for Simulating the Likelihood of Stochastic Differential Equations, The Econometrics Journal, vol. 5, no. 1, pp. 91-103, 2002.

50. B. Øksendal, Stochastic Differential Equations, An Introduction with Applications, Springer, 5th edition, 2000.

51. B. Øksendal, Stochastic Differential Equations: An Introduction with Applications, Springer, New York, 6th edition, 2003.

52. B. Øksendal, An Introduction to Malliavin Calculus with Application to Economics, Lecture Notes, Norwegian School of Economics and Business Administration, 1997.

53. A. Pedersen, A new approach to maximum likelihood estimation for stochastic differential equations based on discrete observations, Scandinavian journal of statistics, vol. 22, no. 1 pp. 55-71, 1995.

54. J. Pitman, M. Yor The two-parameter Poisson Dirichlet distribution derived from a stable sub-ordinator, The Annals of Probability, vol. 25, no. 2, pp. 855-900, 1997.

55. M. Pitt and N. Shephard, Filtering via simulation: Auxiliary particle filters, Journal of the American statistical association, vol. 94, no. 446, pp. 590-599, 1999.

56. A. Rabaoui, N. Viandier, J. Marais, E. Duos, P. Vanheeghe, Dirichlet Process Mixtures for Density Estimation in Dynamic Nonlinear Modeling: Application to GPS Positioning in Urban Canyons, IEEE Transactions on Signal Processing, vol. 60, no. 4, pp. 1638-1655, 2012.

57. A. Ruttor, P. Batz, M. Opper, Approximate Gaussian process inference for the drift function in stochastic differential equations, Advances in Neural Information Processing Systems, pp. 2040-2048, 2013.

58. L. Sánchez, S. Infante, V. Griffin and D. Rey, Spatiotemporal dynamic model and parallelized ensemble Kalman Filter for precipitation data, Brazilian Journal of Probability and Statistics (in press).

59. L. Sánchez, S. Infante, J. Marcano, V. Griffin Polinomial Chaos based on the parallelized ensamble Kalman filter to estimate precipitation states, Statistics, Optimization and Information Computing, vol. 3, no. 1, pp. 79-95, 2015.

60. S. Sarkka, Bayesian filtering and smoothing, Cambridge University Press, 2013.

61. H. Sorenson, D. Alspach, Recursive Bayesian Estimation Using Gaussian Sums, Automatica, vol. 7, no. 4, pp. 465-479, 1971.

62. J. Sethuraman, A constructive definition of Dirichlet priors, Statistica Sinica, pp. 639-650, 1994.

63. Z. Schuss, Nonlinear Filtering and Optimal Phase Tracking Applied, Springer Science \& Business Media, vol. $180,2011$. 
64. M. Stefano, Simulation and inference for stochastic differential equations: with R examples, Springer Science \& Business Media, 2009.

65. M. Stefano, sde: Simulation and Inference for Stochastic Differential Equations, R package version, vol. 2, no. $10,2009$.

66. O. Stramer and M. Bognar, Bayesian inference for irreducible diffusion processes using the pseudo-marginal approach, Bayesian Analysis, vol. 6, no. 2, pp. 231-258, 2011.

67. O. Stramer, M. Bognar and P. Schneider, Bayesian Inference of Discretely Sampled Markov Processes with Closed-Form Likelihood Expansions, The Journal of Financial Econometrics, vol. 8, no. 4, pp. 450-480, 2010.

68. H. Tanizaki, Nonlinear Filters Estimation and Applications, Springer Science \& Business Media, 2013.

69. G. Terejanu, T. Singh, P. Scott A Novel Gaussian Sum Filter Method for Accurate Solution to Nonlinear Filtering Problem, Information Fusion, 2008 11th International Conference on. IEEE, pp. 1-8, 2008.

70. C. Yau, O. Papaspiliopoulos, G. Roberts, C. Holmes, Bayesian non-parametric hidden Markov models with applications in genomics, Journal of the Royal Statistical Society: Series B (Statistical Methodology), vol. 73, no. 1, pp. 37-57, 2011.

71. M. West, Mixtures models, Monte Carlo, Bayesian updating and dynamic models, Computing Science and Statistics, pp. 325-335, 1993. 